University of Wollongong

Research Online

Faculty of Engineering and Information

Faculty of Engineering and Information

Sciences - Papers: Part A

Sciences

$1-1-2013$

\title{
Unstable willmore surfaces of revolution subject to natural boundary conditions
}

Anna Dall'Acqua

Otto-Von-Guericke-Universitat

Klaus Deckelnick

Otto-Von-Guericke-Universitat

Glen Wheeler

University of Wollongong, glenw@uow.edu.au

Follow this and additional works at: https://ro.uow.edu.au/eispapers

Part of the Engineering Commons, and the Science and Technology Studies Commons

Research Online is the open access institutional repository for the University of Wollongong. For further information contact the UOW Library: research-pubs@uow.edu.au 


\title{
Unstable willmore surfaces of revolution subject to natural boundary conditions
}

\begin{abstract}
In the class of surfaces with fixed boundary, critical points of the Willmore functional are naturally found to be those solutions of the Euler-Lagrange equation where the mean curvature on the boundary vanishes. We consider the case of symmetric surfaces of revolution in the setting where there are two families of stable solutions given by the catenoids. In this paper we demonstrate the existence of a third family of solutions which are unstable critical points of the Willmore functional, and which spatially lie between the upper and lower families of catenoids. Our method does not require any kind of smallness assumption, and allows us to derive some additional interesting qualitative properties of the solutions.
\end{abstract}

\section{Keywords}

willmore, boundary, conditions, surfaces, revolution, subject, unstable, natural, ERA2015

Disciplines

Engineering | Science and Technology Studies

\section{Publication Details}

Dall'Acqua, A., Deckelnick, K. \& Wheeler, G. (2013). Unstable willmore surfaces of revolution subject to natural boundary conditions. Calculus of Variations and Partial Differential Equations, 48 (3-4), 293-313. 


\title{
Unstable Willmore surfaces of revolution subject to natural boundary conditions
}

\author{
Anna Dall'Acqua* · Klaus Deckelnick · Glen \\ Wheeler
}

the date of receipt and acceptance should be inserted later

\begin{abstract}
In the class of surfaces with fixed boundary, critical points of the Willmore functional are naturally found to be those solutions of the Euler-Lagrange equation where the mean curvature on the boundary vanishes. We consider the case of symmetric surfaces of revolution in the setting where there are two families of stable solutions given by the catenoids. In this paper we demonstrate the existence of a third family of solutions which are unstable critical points of the Willmore functional, and which spatially lie between the upper and lower families of catenoids. Our method does not require any kind of smallness assumption, and allows us to derive some additional interesting qualitative properties of the solutions.
\end{abstract}

Mathematics Subject Classification (2000) 35J40 - 35B38 · 58E99 - 49J45 · 49Q10

\section{Introduction}

Given a smooth immersed surface $f: \Sigma \rightarrow \mathbb{R}^{3}$, the Willmore functional is defined by

$$
\mathcal{W}(f)=\int_{\Sigma} H^{2} d \mu_{g}
$$

where $g$ is the Riemannian metric induced by the standard Euclidean inner product $\left\langle\cdot, \cdot \cdot\right.$ in $\mathbb{R}^{3}$ along the pull-back of $f, d \mu_{g}=\sqrt{\operatorname{det}(g)} d x$ is the induced surface element, $H=\left(\kappa_{1}+\kappa_{2}\right) / 2$ denotes the mean curvature, and $\kappa_{1}, \kappa_{2}$ are the principal curvatures of $f$. An important feature of the Willmore functional is its invariance under the full Möbius group of $\mathbb{R}^{3}$. (See $[5,26]$, [28, Section 7.3] and Weiner [27].)

A critical point of the Willmore functional is called a Willmore surface and is a solution of the Willmore equation

$$
\Delta H+2 H\left(H^{2}-K\right)=0 \text { on } \Sigma
$$

where $\Delta$ denotes the Laplace-Beltrami operator on $f$ and $K=\kappa_{1} \kappa_{2}$ is the Gauss curvature. In the literature there are many results concerning the existence and regularity of Willmore surfaces. The case where $f$ is a closed immersed surface has been well-studied, and we only mention here the papers $[1,7,16,17,22,24]$. For the case where $f$ is an immersed surface with boundary much less is known. In this setting one must supplement (1) with appropriate boundary conditions. A discussion of the range of possibilities can be found in Nitsche [19]. One possible choice is the Dirichlet boundary conditions

* Corresponding author

Otto-von-Guericke-Universität, Universitätsplatz 2, D-39016 Magdeburg, Germany, Tel: +49 391-67-11993, Fax: +49 391-67-18073,

E-mail: anna.dallacqua@ovgu.de

E-mail: klaus.deckelnick@ovgu.de

E-mail: wheeler@ovgu.de 
where the boundary of the surface and the tangent bundle at the boundary are prescribed. Another is the natural (or Navier) boundary conditions. In this case the position and the mean curvature $H \equiv 0$ are prescribed as boundary data. The latter boundary condition arises naturally from the formula for the first variation (see (4) below). The Navier Willmore boundary value problem is that which we shall study in this paper. For both types of boundary conditions, existence results obtained through perturbative methods can be found in [19,21]. Schätzle in [23] proves the existence of Willmore surfaces satisfying Dirichlet boundary conditions in $\mathbb{S}^{n}$. These are embedded if their Willmore energy is small enough. Uniqueness theorems without symmetry assumptions are due to Palmer [21] and the first author [9]. So far other existence results are known only in the class of embedded surfaces of revolution. It is interesting to study this special case in order to understand the possible qualitative behavior of the solutions and which phenomena may occur. Existence results for Willmore surfaces of revolution subject to Dirichlet and Navier boundary conditions have been obtained in $[10,11,13]$ and $[2,3,12]$ respectively. Before stating our new result we must first fix some notation.

Given a smooth and positive function $u:[-1,1] \rightarrow \mathbb{R}$ we consider the surface of revolution $\Gamma(u)=f_{u}([-1,1] \times[0,2 \pi])$, where $f_{u}$ is the embedding associated to $u$ defined by

$$
f_{u}:(x, \varphi) \mapsto(x, u(x) \cos \varphi, u(x) \sin \varphi), \quad x \in[-1,1], \varphi \in[0,2 \pi] .
$$

The surface $\Gamma(u)$ is said to be generated by $u$. For such a surface the mean curvature and the Gauss curvature are given by

$$
\begin{aligned}
& H(x)=H[u](x)=\frac{1}{2}\left(-\frac{u^{\prime \prime}(x)}{\left(1+u^{\prime}(x)^{2}\right)^{3 / 2}}+\frac{1}{u(x) \sqrt{1+u^{\prime}(x)^{2}}}\right), \quad \text { and } \\
& K(x)=K[u](x)=-\frac{u^{\prime \prime}(x)}{u(x)\left(1+u^{\prime}(x)^{2}\right)^{2}},
\end{aligned}
$$

respectively. The Willmore functional reads

$$
\begin{aligned}
\mathcal{W}(u) & :=\mathcal{W}\left(f_{u}\right) \\
& =\frac{\pi}{2} \int_{-1}^{1}\left(-\frac{u^{\prime \prime}(x)}{\left(1+u^{\prime}(x)^{2}\right)^{3 / 2}}+\frac{1}{u(x) \sqrt{1+u^{\prime}(x)^{2}}}\right)^{2} u(x) \sqrt{1+u^{\prime}(x)^{2}} d x .
\end{aligned}
$$

Given $\alpha>0$, let us denote by $S_{\alpha}$ the set of positive, symmetric functions in $H^{2}(-1,1)$ with boundary value $\alpha$; that is,

$$
S_{\alpha}=\left\{u \in H^{2}(-1,1) \mid u(x)>0, u(x)=u(-x), x \in[-1,1] \text { and } u( \pm 1)=\alpha\right\} .
$$

The first variation of the Willmore functional $\mathcal{W}$ at $u \in S_{\alpha} \cap H^{4}(-1,1)$ in the direction of a function $\varphi \in H^{2}(-1,1) \cap H_{0}^{1}(-1,1)$ is given by (cf. [12, Lemma 6])

$$
\left.\frac{d}{d t} \mathcal{W}(u+t \varphi)\right|_{t=0}=-2 \pi\left[H \frac{u \varphi^{\prime}}{1+\left(u^{\prime}\right)^{2}}\right]_{-1}^{1}-2 \pi \int_{-1}^{1} u \varphi\left(\Delta H+2 H\left(H^{2}-K\right)\right) d x .
$$

Critical points then solve the following Navier boundary value problem:

$$
\begin{aligned}
& \Delta H+2 H\left(H^{2}-K\right)=0 \text { in }(-1,1), \\
& u( \pm 1)=\alpha, H( \pm 1)=0 .
\end{aligned}
$$

Note that (5) is equivalent to (cf. [10, Section 2.1])

$$
\begin{aligned}
& \frac{1}{u(x) \sqrt{1+u^{\prime}(x)^{2}}} \frac{d}{d x}\left(\frac{u(x)}{\sqrt{1+u^{\prime}(x)^{2}}} H^{\prime}(x)\right) \\
& \quad+\frac{1}{2} H(x)\left(\frac{u^{\prime \prime}(x)}{\left(1+u^{\prime}(x)^{2}\right)^{\frac{3}{2}}}+\frac{1}{u(x) \sqrt{1+u^{\prime}(x)^{2}}}\right)^{2}=0, \quad x \in(-1,1) .
\end{aligned}
$$


By also expressing $H$ in terms of $u$ one observes that (5) is a highly nonlinear ODE of fourth order. Furthermore, the boundary conditions $H( \pm 1)=0$ are nonlinear as well. Particular solutions of (5)-(6) are given by the family of catenaries

$$
u_{b}(x)=\frac{1}{b} \cosh (b x)
$$

as long as the equation $\alpha=u_{b}( \pm 1)=\frac{\cosh (b)}{b}$ has a positive solution. There is a critical boundary value $\alpha^{*}$ below which catenaries cease to exist, given by

$$
\alpha^{*}=\inf _{y \in(0, \infty)} \frac{\cosh (y)}{y}=\frac{\cosh \left(b_{0}\right)}{b_{0}} \simeq 1.5088
$$

with $b_{0}=1.1996 \ldots$ satisfying $\cosh \left(b_{0}\right)=b_{0} \sinh \left(b_{0}\right)$. For $\alpha>\alpha^{*}$ the equation $\alpha=\frac{\cosh (b)}{b}$ has precisely two solutions $0<b_{1}(\alpha)<b_{0}<b_{2}(\alpha)<\infty$, and since $H\left[u_{b_{1}}\right] \equiv H\left[u_{b_{2}}\right] \equiv 0$, the functions $u_{b_{1}}$ and $u_{b_{2}}$ are solutions of (5)-(6). As $\mathcal{W}(u) \geq 0$ and $\mathcal{W}\left(u_{b_{1}}\right)=\mathcal{W}\left(u_{b_{2}}\right)=0$, these functions are global minimisers of $\mathcal{W}$ in the class $S_{\alpha}$. It is natural to expect the existence of a third critical point of $\mathcal{W}$ between the two catenaries. By carrying out a bifurcation analysis it was shown in [12] that $\left(b_{0}, u_{b_{0}}\right)$ is the unique bifurcation point for (5)-(6) on the branch $b \mapsto\left(b, u_{b}\right)$ in the class of symmetric solutions; in particular one has:

Theorem 1 ([12, Theorem 1.3]) There exists an $\varepsilon>0$ such that for $\alpha \in\left(\alpha^{*}, \alpha^{*}+\varepsilon\right)$ the boundary value problem (5)-(6) has at least three solutions in $S_{\alpha}$. One of these is not a minimal surface.

In order to extend this result for larger values of $\alpha$ it is natural to first attempt a straightforward adaptation of classical mountain pass theory. The min-max characterisation of the Palais-Smale limit would then automatically imply instability. Such a strategy was successfully carried out by Struwe (see [25] and the references therein) to prove the existence of unstable solutions to the Plateau problem via an adaptation of classical Ljusternik-Schnirelmann theory. Palais-Smale sequences for the Willmore functional have been recently investigated in [4], where it is proven that local PalaisSmale sequences that are uniformly bounded in $W^{2,2} \cap W^{1, \infty}$ possess a convergent subsequence. The limit is shown to satisfy a system of differential equations which, under a smallness condition on the energy, is equivalent to the constrained Willmore equation. The indication from [4] is thus that for a Palais-Smale approach to be successful, one typically requires some kind of energy assumption. By following a different approach we are able to avoid any smallness assumption in our case and obtain the following global result.
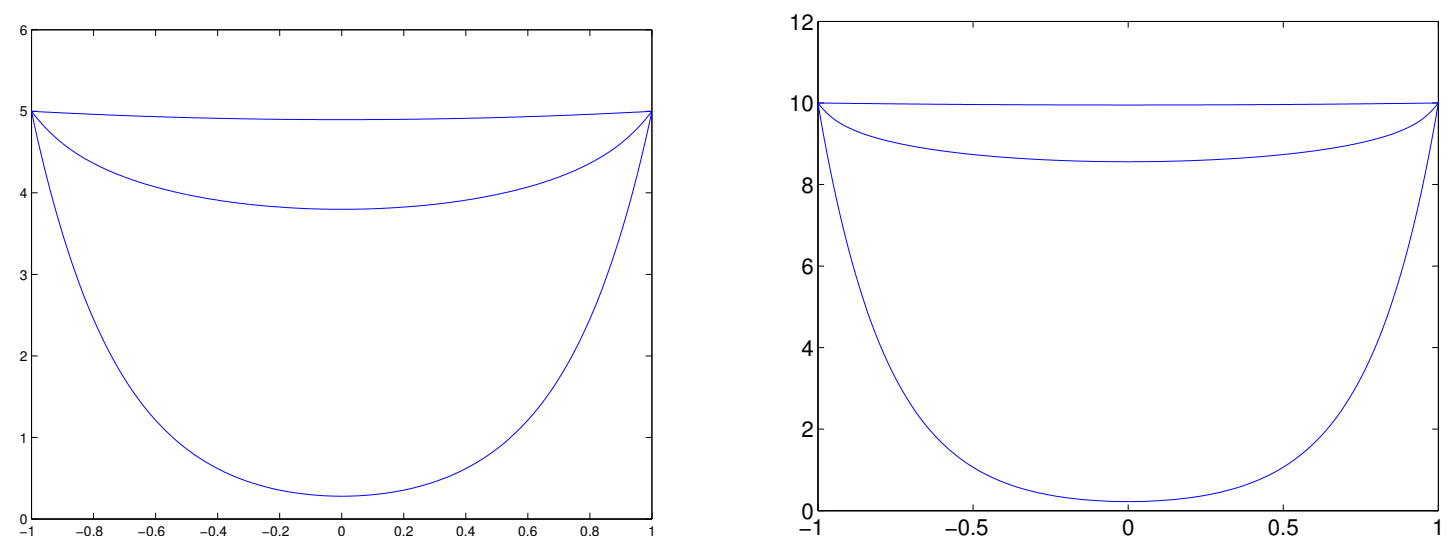

Fig. 1: Numerically calculated unstable solutions between the two catenaries for $\alpha=5$ (left) and $\alpha=10$ (right). (See also Remark 5.) 
Theorem 2 For every $\alpha>\alpha^{*}$ the boundary value problem (5)-(6) has, in addition to the two catenaries $u_{b_{1}}$ and $u_{b_{2}}$, a third smooth solution $u \in S_{\alpha}$ which is unstable in the sense that $u$ is not a local minimum of $\mathcal{W}$ in $S_{\alpha}$. Furthermore

(i) $H[u]<0$ in $(-1,1)$;

(ii) $u$ and $H[u]$ are strictly convex in $[-1,1]$;

(iii) $u_{b_{2}}(x)<u(x)<u_{b_{1}}(x)$ for $x \in(-1,1)$.

To our knowledge, this is the first existence result for unstable Willmore surfaces satisfying prescribed boundary conditions. A sufficient condition for unstable closed Willmore surfaces is given in [20] together with some examples. (See also [18].)

The proof of Theorem 2 essentially consists of two steps. In the first step, we show that the Willmore equation is equivalent to certain singular first order ODEs for the mean curvature $H[u]$.

Theorem 3 Let $u:[-1,1] \rightarrow \mathbb{R}$ be a smooth, positive, symmetric function. Then $u$ is a solution of (5) if and only if for all $x \in[-1,1]$

$$
\frac{H^{\prime}(x) u(x)}{1+u^{\prime}(x)^{2}}\left(u(x)-x u^{\prime}(x)\right)-\frac{H(x) u(x)\left(x+u(x) u^{\prime}(x)\right)}{\sqrt{1+u^{\prime}(x)^{2}}}\left(H(x)-\frac{1}{u(x) \sqrt{1+u^{\prime}(x)^{2}}}\right)=0 .
$$

Furthermore, if $u$ is a solution of (5) then there exists a constant $C$ such that for all $x \in[-1,1]$

$$
-\frac{H^{\prime}(x) u(x) u^{\prime}(x)}{1+u^{\prime}(x)^{2}}-\frac{u(x) H(x)^{2}}{\sqrt{1+u^{\prime}(x)^{2}}}+\frac{H(x)}{1+u^{\prime}(x)^{2}}=C .
$$

The converse holds provided $u$ is not identically constant on $[-1,1]$.

The proof of this result will be given in Section 2 and relies on the scale and translation invariance of the Willmore functional. By evaluating (8) at $x=1$ one readily confirms that a smooth, symmetric solution of $(5)$ with $u( \pm 1)= \pm u^{\prime}( \pm 1)$ satisfies

$$
H( \pm 1)=0 \quad \text { or } \quad H( \pm 1)=\frac{1}{u(1) \sqrt{1+u^{\prime}(1)^{2}}}
$$

This observation allows us in the second step to obtain a solution of (5)-(6) by constructing a symmetric solution of $(5)$ with $u( \pm 1)=\alpha, u^{\prime}( \pm 1)= \pm \alpha$ in such a way that the case $H( \pm 1)=\frac{1}{\alpha \sqrt{1+\alpha^{2}}}$ is excluded. In this manner we are led to consider a particular Dirichlet boundary value problem (see (15)) which is accessible to the direct method of the calculus of variations. We approach this problem in Section 3 by minimising $\mathcal{W}$ over the sets

$$
\tilde{N}_{\alpha, \beta}:=\left\{u \in S_{\alpha} \cap C^{1,1}([-1,1]): u^{\prime}(-1)=\beta, u^{\prime}(x) \leq \alpha \text { for all } x \in[0,1]\right\}
$$

for $\beta>-\alpha$. The desired solution $u$ is then obtained as the limit of minimisers when $\beta \searrow-\alpha$. Perhaps surprisingly, the constraint $u^{\prime} \leq \alpha$ ensures that $u$ is convex which in turn implies that $H( \pm 1)=0$. At the same time this method yields the instability of our solution by exploiting the strict monotonicity of the map

$$
\beta \mapsto \inf _{v \in \tilde{N}_{\alpha, \beta}} \mathcal{W}(v)
$$

Such monotonicity properties were used in [2] to prove the existence of solutions to the Navier boundary value problem for $\alpha<\alpha^{*}$. The qualitative properties $(i)-(i i i)$ in Theorem 2 are finally obtained by combining the minimising property of $u$ with (8) and (9). 


\section{Proof of Theorem 3}

An important feature of the Willmore functional is its invariance with respect to conformal transformations. It is natural to expect that this large class of invariances yields extra information at the boundary, in the spirit of the Pohozaev identity. In the case under consideration, we can obtain quite a lot of additional information. For this purpose it is useful to derive the first variation of $\mathcal{W}$.

Given a compact surface $\Sigma$ with boundary and a sufficiently smooth immersion $f: \Sigma \rightarrow \mathbb{R}^{3}$ we denote by $\mathbf{W}(f):=\left(\Delta H+2 H\left(H^{2}-K\right)\right) \boldsymbol{\nu}$ the Euler-Lagrange operator of $\mathcal{W}$ in $L^{2}$ at $f$, with $\boldsymbol{\nu}$ the exterior unit normal vectorfield, and use $\phi^{\perp}:=\langle\phi, \nu\rangle \nu$ to denote the normal projection of a vectorfield $\phi$. We also use the notation $\mathbf{H}:=-H \boldsymbol{\nu}$ to denote the mean curvature vector.

Lemma 4 ([15, Theorem 2.1]) Let $\Sigma$ be a compact surface with boundary, $t_{0} \in \mathbb{R}$, and $\delta>0$. For a smooth variation $h: \Sigma \times\left(t_{0}-\delta, t_{0}+\delta\right) \rightarrow \mathbb{R}^{3}$ with $\phi=\partial_{t} h$ we have

$$
\frac{d}{d t} \mathcal{W}(h(\cdot, t))=-\int_{\Sigma}\left(\left\langle\mathbf{W}(h(\cdot, t)), \phi^{\perp}\right\rangle+\nabla^{*} \omega\right) d \mu_{g}
$$

Here, given a frame $\left\{\tau_{i}\right\}_{i=1}^{2}$ on $T \Sigma$, the one-form $\omega$ is defined by

$$
\omega\left(\tau_{i}\right)=2\left\langle\phi,\left(\partial_{i} \mathbf{H}\right)^{\perp}\right\rangle-\partial_{i}\langle\phi, \mathbf{H}\rangle-|\mathbf{H}|^{2}\left\langle\phi, \partial_{i} h\right\rangle, \text { and } \nabla^{*} \omega=-\sum_{i, j=1}^{2} g^{i j} \nabla_{i} \omega\left(\tau_{j}\right)
$$

where $\partial_{i}$ is the derivative along $\tau_{i}, \nabla_{i}$ is the covariant derivative along $\tau_{i}$, and $g^{i j}=\left(g^{-1}\right)_{i j}$ are elements of the inverse of the induced metric $g$.

Choosing $h$ in (10) according to the invariances of the Willmore functional allows us now to carry out the

Proof (Proof of Theorem 3) We first prove that every smooth, positive, symmetric solution of (5) satisfies (8) and (9). The opposite implications will be discussed together afterwards.

Consider the cylinder $\Sigma=[-1,1] \times[0,2 \pi]$, where we have identified 0 and $2 \pi$, and set $f_{u}: \Sigma \rightarrow \mathbb{R}^{3}$ to be the embedding associated to $u$ as in (2). As $u$ satisfies (5), $f_{u}$ is clearly a Willmore surface and $\mathbf{W}\left(f_{u}\right) \equiv 0$. Let $x_{1}, x_{2} \in[-1,1]$ with $x_{1}<x_{2}$, and consider the cylinder $\tilde{\Sigma}=\left[x_{1}, x_{2}\right] \times[0,2 \pi]$, where we have again identified 0 and $2 \pi$. Let $h: \tilde{\Sigma} \times\left(t_{0}-\delta, t_{0}+\delta\right) \rightarrow \mathbb{R}^{3}$ be a family of sufficiently smooth immersions as in Lemma 4 satisfying $h\left(\cdot, t_{0}\right)=f_{u}(\cdot)$. Denote by $\partial_{1} \tilde{\Sigma}$ and by $\partial_{2} \tilde{\Sigma}$ the left and right boundaries of $\tilde{\Sigma}$ respectively, so that

$$
\partial \tilde{\Sigma}=\partial_{1} \tilde{\Sigma} \cup \partial_{2} \tilde{\Sigma} \text {, with } \partial_{j} \tilde{\Sigma} \text { the circle given by } \partial_{j} \tilde{\Sigma}=\left\{x_{j}\right\} \times[0,2 \pi], j=1,2 \text {. }
$$

We denote by $\eta_{j}$ the interior unit conormal of $\partial_{j} \tilde{\Sigma}$. The embedding (2) induces a global orthonormal frame $\left\{\tau_{x}, \tau_{\varphi}\right\}$ for the tangent bundle over $\Sigma$, where

$$
\tau_{x}=\frac{1}{\sqrt{1+u^{\prime}(x)^{2}}} \partial_{x}, \text { and } \tau_{\varphi}=\frac{1}{u(x)} \partial_{\varphi}
$$

Note that via the embedding this induces the following frame

$$
\begin{aligned}
& \left\{\tau_{x} h\left((x, \varphi), t_{0}\right), \tau_{\varphi} h\left((x, \varphi), t_{0}\right)\right\}=\left\{\tau_{x} f_{u}(x, \varphi), \tau_{\varphi} f_{u}(x, \varphi)\right\} \\
& \quad=\left\{\frac{1}{\sqrt{1+u^{\prime}(x)^{2}}}\left(1, u^{\prime}(x) \cos \varphi, u^{\prime}(x) \sin \varphi\right),(0,-\sin \varphi, \cos \varphi)\right\}
\end{aligned}
$$

of the tangent space at $(x, u(x) \cos \varphi, u(x) \sin \varphi)$. In these coordinates $\eta_{1}(\varphi)=\tau_{x} h\left(\left(x_{1}, \varphi\right), t_{0}\right)$, and by symmetry $\eta_{2}(\varphi)=-\tau_{x} h\left(\left(x_{2}, \varphi\right), t_{0}\right)$. Since $h\left(\cdot, t_{0}\right)=f_{u}(\cdot)$ is a Willmore surface, Lemma 4 
combined with Stokes' theorem (note that $\omega$ is a 1-form) yields

$$
\begin{aligned}
\left.\frac{d}{d t} \mathcal{W}(h)\right|_{t=t_{0}} & =\int_{\tilde{\Sigma}} \sum_{i, j=1}^{2} g^{i j} \nabla_{i} \omega_{j} d \mu_{g}=-\int_{\tilde{\Sigma}} \nabla^{*} \omega d \mu_{g} \\
& =\int_{\partial_{1} \tilde{\Sigma}} \omega\left(\eta_{1}\right) d \mu_{\partial_{1} g}+\int_{\partial_{2} \tilde{\Sigma}} \omega\left(\eta_{2}\right) d \mu_{\partial_{2} g} \\
& =\int_{\partial_{1} \tilde{\Sigma}} \omega\left(\tau_{x}\right) d \mu_{\partial_{1} g}-\int_{\partial_{2} \tilde{\Sigma}} \omega\left(\tau_{x}\right) d \mu_{\partial_{2} g}
\end{aligned}
$$

where $d \mu_{\partial_{j} g}$ is the induced metric on $\partial_{j} \tilde{\Sigma}$. Using the explicit expression for $\tau_{x}$ given above and taking note of the definition of $\omega$ from Lemma 4 , we find

$$
\begin{aligned}
\left.\frac{d}{d t} \mathcal{W}(h)\right|_{t=t_{0}} & \int_{\partial_{1} \tilde{\Sigma}}\left[2\left\langle\phi,\left(\partial_{x} \mathbf{H}\right)^{\perp}\right\rangle-\partial_{x}\langle\phi, \mathbf{H}\rangle-|\mathbf{H}|^{2}\left\langle\phi, \partial_{x} f_{u}\right\rangle\right] \frac{1}{\sqrt{1+u^{\prime}(x)^{2}}} d \mu_{\partial_{1} g} \\
& -\int_{\partial_{2} \tilde{\Sigma}}\left[2\left\langle\phi,\left(\partial_{x} \mathbf{H}\right)^{\perp}\right\rangle-\partial_{x}\langle\phi, \mathbf{H}\rangle-|\mathbf{H}|^{2}\left\langle\phi, \partial_{x} f_{u}\right\rangle\right] \frac{1}{\sqrt{1+u^{\prime}(x)^{2}}} d \mu_{\partial_{2} g} \\
= & \left.\int_{0}^{2 \pi}\left[2\left\langle\phi,\left(\partial_{x} \mathbf{H}\right)^{\perp}\right\rangle-\partial_{x}\langle\phi, \mathbf{H}\rangle-|\mathbf{H}|^{2}\left\langle\phi, \partial_{x} f_{u}\right\rangle\right] \frac{u(x)}{\sqrt{1+u^{\prime}(x)^{2}}} d \varphi\right|_{x=x_{1}} \\
& -\left.\int_{0}^{2 \pi}\left[2\left\langle\phi,\left(\partial_{x} \mathbf{H}\right)^{\perp}\right\rangle-\partial_{x}\langle\phi, \mathbf{H}\rangle-|\mathbf{H}|^{2}\left\langle\phi, \partial_{x} f_{u}\right\rangle\right] \frac{u(x)}{\sqrt{1+u^{\prime}(x)^{2}}} d \varphi\right|_{x=x_{2}} .
\end{aligned}
$$

In order to proceed we need to choose the direction of the variation $\phi$. It is natural to consider variations parallel to the generators of the Möbius group of $\mathbb{R}^{3}$, since for such $\phi$ the Willmore functional is invariant and the left hand side of (11) is automatically zero. For the purposes of proving (8) and (9) it will be enough to consider the invariance of $\mathcal{W}$ under scaling and translation respectively.

We begin with the scale invariance of $\mathcal{W}$. Consider the family of immersions $h((x, \varphi), t)=$ $t(x, u(x) \cos (\varphi), u(x) \sin (\varphi))$ and take $t_{0}=1$. Then

$$
\phi(x, \varphi)=(x, u(x) \cos \varphi, u(x) \sin \varphi)=f_{u}(x, \varphi) .
$$

The exterior unit normal $\boldsymbol{\nu}$ is given by

$$
\boldsymbol{\nu}(x, \varphi)=\frac{1}{\sqrt{1+u^{\prime}(x)^{2}}}\left(-u^{\prime}(x), \cos \varphi, \sin \varphi\right),
$$

and $\left(\partial_{x} \mathbf{H}\right)^{\perp}(x, \varphi)=\left\langle\left(\partial_{x} \mathbf{H}\right)(x, \varphi), \boldsymbol{\nu}(x, \varphi)\right\rangle \boldsymbol{\nu}(x, \varphi)=-H^{\prime}(x) \boldsymbol{\nu}(x, \varphi)$. We thus have for the first term

$$
2\left\langle\phi(x, \varphi),\left(\partial_{x} \mathbf{H}\right)^{\perp}(x, \varphi)\right\rangle=-2 H^{\prime}(x)\left\langle f_{u}(x, \varphi), \boldsymbol{\nu}(x, \varphi)\right\rangle=2 H^{\prime}(x) \frac{x u^{\prime}(x)-u(x)}{\sqrt{1+u^{\prime}(x)^{2}}} .
$$

Note that the final expression no longer depends on $\varphi$. Keeping in mind (3), a direct computation simplifies the second term as

$$
\begin{aligned}
-\partial_{x} & \langle\phi(x, \varphi), \mathbf{H}(x, \varphi)\rangle \\
& =-\partial_{x}\left(-\frac{H(x)}{\sqrt{1+u^{\prime}(x)^{2}}}\left(-x u^{\prime}(x)+u(x)\right)\right) \\
& =-H^{\prime}(x) \frac{x u^{\prime}(x)-u(x)}{\sqrt{1+u^{\prime}(x)^{2}}}+2 H^{2}(x)\left(x+u(x) u^{\prime}(x)\right)-H(x) \frac{x+u(x) u^{\prime}(x)}{u(x) \sqrt{1+u^{\prime}(x)^{2}}} .
\end{aligned}
$$


For the third term we have

$$
-|\mathbf{H}|(x, \varphi)^{2}\left\langle\phi(x, \varphi), \partial_{x} f_{u}(x, \varphi)\right\rangle=-H(x)^{2}\left(x+u(x) u^{\prime}(x)\right) .
$$

As $h$ is a continuous family of rescalings of $f_{u}$, the scale invariance of $\mathcal{W}$ implies that the left hand side of (11) vanishes. Inserting the equalities computed above allows us to write down the right hand side of (11) explicitly. We obtain

$$
\begin{aligned}
0=2 \pi u(x)\left(\frac{H^{\prime}(x)}{1+u^{\prime}(x)^{2}}\left(u(x)-x u^{\prime}(x)\right)-\right. & \frac{H(x)^{2}}{\sqrt{1+u^{\prime}(x)^{2}}}\left(x+u(x) u^{\prime}(x)\right) \\
& \left.+\frac{H(x)}{u(x)\left(1+u^{\prime}(x)^{2}\right)}\left(x+u(x) u^{\prime}(x)\right)\right)\left.\right|_{x_{1}} ^{x_{2}} .
\end{aligned}
$$

Clearly, as $u^{\prime}(0)=0$ and $H^{\prime}(0)=0$ equation (8) holds at $x=0$. Formula (8) follows for $x>0$ by taking $x_{2}=x$ and $x_{1}=0$ in (12), while taking $x_{1}=-x$ and $x_{2}=0$ in (12) implies (8) for $x<0$.

The case of translations gives equation (9) in a similar manner. Let us consider the family of immersions $h((x, \varphi), t)=(x, u(x) \cos (\varphi), u(x) \sin (\varphi))+t(1,0,0)$ with $t_{0}=0$. Then $\phi(x, \varphi)=(1,0,0)$. We have for the first term

$$
2\left\langle\phi(x, \varphi),\left(\partial_{x} \mathbf{H}\right)^{\perp}(x, \varphi)\right\rangle=2 \frac{u^{\prime}(x) H^{\prime}(x)}{\sqrt{1+u^{\prime}(x)^{2}}} .
$$

The second term becomes

$$
\begin{aligned}
-\partial_{x}\langle\phi(x, \varphi), \mathbf{H}(x, \varphi)\rangle & =-\partial_{x}\left(\frac{u^{\prime}(x) H(x)}{\sqrt{1+u^{\prime}(x)^{2}}}\right) \\
=- & \frac{H^{\prime}(x) u^{\prime}(x)}{\sqrt{1+u^{\prime}(x)^{2}}}-\frac{H(x)}{u(x) \sqrt{1+u^{\prime}(x)^{2}}}+2 H(x)^{2} .
\end{aligned}
$$

For the third term we have

$$
-|\mathbf{H}|(x, \varphi)^{2}\left\langle\phi(x, \varphi), \partial_{x} f_{u}(x, \varphi)\right\rangle=-H(x)^{2} .
$$

As $h$ is a continuous family of translations, the translation invariance of $\mathcal{W}$ combined with the above formulae simplify (11) to

$$
-\frac{H^{\prime}(x) u(x) u^{\prime}(x)}{1+u^{\prime}(x)^{2}}-\frac{u(x) H(x)^{2}}{\sqrt{1+u^{\prime}(x)^{2}}}+\left.\frac{H(x)}{1+u^{\prime}(x)^{2}}\right|_{x_{1}} ^{x_{2}}=0 .
$$

Since $x_{1}, x_{2} \in[-1,1]$ are arbitrary, it follows that there exists a constant $C$ such that

$$
-\frac{H^{\prime}(x) u(x) u^{\prime}(x)}{1+u^{\prime}(x)^{2}}-\frac{u(x) H(x)^{2}}{\sqrt{1+u^{\prime}(x)^{2}}}+\frac{H(x)}{1+u^{\prime}(x)^{2}}=C, \quad \text { for } x \in[-1,1],
$$

which is (9).

Let us now show that every solution of (8) satisfies (5). Denoting the left hand side of (8) by $L[u](x)$, a long but straightforward computation shows that

$$
0=\frac{d}{d x} L[u](x)=u(x)\left(u(x)-x u^{\prime}(x)\right)\left(\Delta H+2 H\left(H^{2}-K\right)\right)(x), x \in[-1,1] .
$$

Let $E=\left\{x \in[-1,1] \mid u(x)-x u^{\prime}(x)=0\right\}$. Clearly, (5) holds at all points $x \in[-1,1] \backslash E$. Due to the smoothness of $u$, it is sufficient to show that $[-1,1] \backslash E$ is dense in $[-1,1]$. If this were not the case, there would be an open interval $\left(x_{0}-\delta, x_{0}+\delta\right)$ such that $u(x)-x u^{\prime}(x)=0$ in $\left(x_{0}-\delta, x_{0}+\delta\right) \cap[-1,1]=: I$. This implies $u(x)=a x$ in $I$ for some constant $a$, and therefore

$$
L[u](x) \equiv \frac{1}{4 a \sqrt{1+a^{2}}} \neq 0 \text { in } I,
$$


which contradicts (8).

Finally we show that a solution to (9) that is not identically constant in $[-1,1]$ satisfies (5). Denoting the left hand side of (9) by $M[u](x)$, a computation shows that

$$
0=\frac{d}{d x} M[u](x)=u(x) u^{\prime}(x)\left(\Delta H+2 H\left(H^{2}-K\right)\right)(x), x \in[-1,1] .
$$

Let $E=\left\{x \in[-1,1] \mid u^{\prime}(x)=0\right\}$. Note that $E \subsetneq[-1,1]$ since $u$ is not identically constant on $[-1,1]$. Clearly, (5) is satisfied at $x \in[-1,1] \backslash E$. As above, it is sufficient to show that $[-1,1] \backslash E$ is dense in $[-1,1]$. If this were not the case, there would be an open interval $I \subset E$ such that there exists $x^{*} \in \partial I$ that is an accumulation point for $[-1,1] \backslash E$. Then by continuity (5) is satisfied at $x^{*}$. On the other hand, since $I \subset E$ there exists a positive constant $a$ such that $u(x) \equiv a$ for $x \in \bar{I}$ and therefore

$$
\left(\Delta H+2 H\left(H^{2}-K\right)\right)\left(x^{*}\right)=\frac{1}{4 a^{3}} \neq 0,
$$

a contradiction. This finishes the proof of Theorem 3 .

Remark 1 In the proof of Theorem 3 we have used the translation invariance only in the direction $(1,0,0)$. In other directions the integrand retains a dependence on the angle $\varphi$ and hence no interesting equation can be derived. This also occurs if we use the invariance with respect to rotations. It seems that we cannot use the invariance with respect to inversions since we cannot write it as a continuous transformation.

Remark 2 One can also derive formulas (8) and (9) from (5) by integrating (13) and (14) on intervals $\left(x_{1}, x_{2}\right)$. The surprising fact about (13) and (14) is that the multiplication of the Willmore operator by suitable factors enables us to recognise a derivative. However it is difficult to guess the right form of these factors in advance. We have therefore chosen to use the invariances of the Willmore functional to prove (8) and (9) because this approach seems to be more natural and easier to generalise.

Theorem 5 Let $u:[-1,1] \rightarrow \mathbb{R}$ be a smooth, positive, symmetric solution of (5) satisfying $u(1)=$ $u^{\prime}(1)$. Then,

$$
H(1)=0 \quad \text { or } \quad H(1)=\frac{1}{u(1) \sqrt{1+u^{\prime}(1)^{2}}} .
$$

Proof By Theorem $3 u$ satisfies (8) for all $x \in[-1,1]$. Taking $x=1$ in (8) and using that $u(1)=u^{\prime}(1)$ we obtain

$$
H(1) u(1) \sqrt{1+u^{\prime}(1)^{2}}\left(\frac{1}{u(1) \sqrt{1+u^{\prime}(1)^{2}}}-H(1)\right)=0,
$$

from which the claim follows.

Corollary 6 Let $\alpha>\alpha^{*}$ and $u:[-1,1] \rightarrow \mathbb{R}$ be a smooth, positive, symmetric, convex solution of the Dirichlet Willmore boundary value problem

$$
\left\{\begin{array}{l}
\Delta H+2 H\left(H^{2}-K\right)=0 \text { in }(-1,1) \\
u( \pm 1)=\alpha, \text { and } u^{\prime}( \pm 1)= \pm \alpha .
\end{array}\right.
$$

Then $u$ solves (5)-(6) and $\Gamma(u)$ is not a minimal surface.

Proof Since $u$ is convex we have $u^{\prime \prime}(x) \geq 0$ and hence

$$
H(1)=\frac{1}{2}\left(-\frac{u^{\prime \prime}(1)}{\left(1+u^{\prime}(1)^{2}\right)^{3 / 2}}+\frac{1}{u(1) \sqrt{1+u^{\prime}(1)^{2}}}\right) \leq \frac{1}{2 u(1) \sqrt{1+u^{\prime}(1)^{2}}} .
$$

Theorem 5 then implies that $H(1)=0$. Finally, if $\Gamma(u)$ were a minimal surface, i.e. $H \equiv 0$, then [8, Proposition 5.11] would imply that $u$ is a catenary. This is impossible due to $\alpha>\alpha^{*}$ and the boundary values of $u$ and $u^{\prime}$. 
In the following proposition we collect some further consequences of Theorem 3 . We shall use (16) to prove that $H[u]<0$ in $(-1,1)$, while we have included (17) because we feel that such a result is of independent interest.

Proposition 7 Let $u:[-1,1] \rightarrow \mathbb{R}$ be a smooth, positive, symmetric solution of (5). Then $H[u]$ satisfies

$$
u^{2}(x) H^{\prime}(x)+C\left(x+u(x) u^{\prime}(x)\right)=0 .
$$

Furthermore, $u$ is a solution of the second order differential equation

$$
-\frac{u(x)^{2} u^{\prime \prime}(x)}{\left(1+u^{\prime}(x)^{2}\right)^{3 / 2}}-\frac{u(x)}{\sqrt{1+u^{\prime}(x)^{2}}}+C\left(u(x)^{2}+x^{2}\right)=D, \quad \text { in }[-1,1] .
$$

Here $C$ is as in (9) and $D$ is a constant of integration.

Proof The differential equation (16) is obtained from Theorem 3 by observing that (8) can be rewritten as

$$
\left(-\frac{H^{\prime}(x) u(x) u^{\prime}(x)}{1+u^{\prime}(x)^{2}}-\frac{u(x) H(x)^{2}}{\sqrt{1+u^{\prime}(x)^{2}}}+\frac{H(x)}{1+u^{\prime}(x)^{2}}\right)\left(x+u(x) u^{\prime}(x)\right)+H^{\prime}(x) u(x)^{2}=0
$$

and using (9). The second statement in the claim follows from the first using

$$
\frac{d}{d x}\left[u(x)^{2} H(x)\right]=u(x)^{2} H^{\prime}(x)+\frac{d}{d x} \frac{u(x)}{\sqrt{1+u^{\prime}(x)^{2}}}
$$

together with the fact that $x+u(x) u^{\prime}(x)=\frac{d}{d x}\left(\frac{1}{2}\left(x^{2}+u(x)^{2}\right)\right)$, integrating once, and using (3).

\section{Existence of a convex solution to (15)}

In view of Corollary 6 we obtain a solution of (5)-(6) with $H \not \equiv 0$ by constructing a convex solution of the Dirichlet Willmore boundary value problem (15). For later purposes it is convenient to consider the family of Dirichlet boundary value problems

$$
\left\{\begin{array}{l}
\Delta H+2 H\left(H^{2}-K\right)=0 \text { in }(-1,1) \\
u( \pm 1)=\alpha, \text { and } u^{\prime}( \pm 1)=\mp \beta
\end{array}\right.
$$

for boundary slopes $\beta \in\left[-\alpha,-\sinh \left(b_{1}(\alpha)\right)\right)$. As in the introduction, we denote by $b_{1}=b_{1}(\alpha), b_{2}=$ $b_{2}(\alpha)$ with $0<b_{1}(\alpha)<b_{0}<b_{2}(\alpha)<\infty$ the two solutions of the equation $\alpha=\frac{\cosh (b)}{b}$. Using the properties of the function $y \mapsto \frac{\cosh (y)}{y}$ one sees that

$$
\frac{\cosh (y)}{y}<\alpha \quad \Longleftrightarrow \quad y \in\left(b_{1}(\alpha), b_{2}(\alpha)\right) .
$$

For $\beta \in\left[-\alpha,-\sinh \left(b_{1}(\alpha)\right)\right)$ we have

$$
\sinh \left(b_{1}\right)<-\beta \leq \alpha=\frac{\cosh \left(b_{2}\right)}{b_{2}}<\sinh \left(b_{2}\right),
$$

so that in the parameter range we consider the two catenaries $u_{b_{1}}, u_{b_{2}}$ are not solutions of (18). Next, let us introduce

$$
\alpha_{\beta}:=\frac{\sqrt{1+\beta^{2}}}{\operatorname{arcsinh}(-\beta)} .
$$

Note that $(20)$ implies that $\operatorname{arcsinh}(-\beta) \in\left(b_{1}(\alpha), b_{2}(\alpha)\right)$ so that $(19)$ with $y=\operatorname{arcsinh}(-\beta)$ implies

$$
\alpha>\alpha_{\beta}, \quad \beta \in\left[-\alpha,-\sinh \left(b_{1}(\alpha)\right)\right) .
$$


Existence of solutions to (18) for arbitrary $\alpha>0$ and $\beta \in \mathbb{R}$ has been obtained in [11] by minimizing over suitable subsets of $H^{2}(-1,1)$ the "hyperbolic Willmore functional"

$$
\mathcal{W}_{h}(u)=\int_{-1}^{1}\left(\frac{u(x) u^{\prime \prime}(x)}{\left(1+u^{\prime}(x)^{2}\right)^{3 / 2}}+\frac{1}{\sqrt{1+u^{\prime}(x)^{2}}}\right)^{2} \frac{\sqrt{1+u^{\prime}(x)^{2}}}{u(x)} d x,
$$

which is the elastic energy of the graph of $u$ as a curve in the hyperbolic half-plane. Motivated by the theory developed in [11, Section 4.2.3] we introduce the following set:

$$
\tilde{N}_{\alpha, \beta}:=\left\{u \in S_{\alpha} \cap C^{1,1}([-1,1]) \mid u^{\prime}(-1)=\beta, u^{\prime}(x) \leq \alpha \text { for all } x \in[0,1]\right\} .
$$

As we shall see later, the constraint on $u^{\prime}$ will ensure the convexity of our solution. In addition we have for all $u \in \tilde{N}_{\alpha, \beta}$

$$
\alpha-u(x)=\int_{x}^{1} u^{\prime}(x) d x \leq \alpha(1-x), \quad x \in[0,1] .
$$

and therefore

$$
u(x) \geq \alpha x, \quad x \in[0,1] .
$$

In view of $(21)$ and the fact that $-\beta \leq \alpha$, we see that the function

$$
\bar{u}(x):=\frac{1}{b} \cosh (b x)+\alpha-\alpha_{\beta}, \quad \text { with } b=\operatorname{arcsinh}(-\beta)
$$

belongs to $\tilde{N}_{\alpha, \beta}$, so that this set is not empty. Let

$$
\tilde{M}_{\alpha, \beta}:=\inf _{u \in \tilde{N}_{\alpha, \beta}} \mathcal{W}(u)
$$

As observed by Pinkall and Bryant-Griffiths [6,14], the Willmore functional and the hyperbolic Willmore functional are related by the identity

$$
\mathcal{W}(u)=\frac{\pi}{2} \mathcal{W}_{h}(u)-\left.2 \pi \frac{u^{\prime}(x)}{\sqrt{1+u^{\prime}(x)^{2}}}\right|_{-1} ^{1}
$$

so that in particular

$$
\mathcal{W}(u)=\frac{\pi}{2} \mathcal{W}_{h}(u)+4 \pi \frac{\beta}{\sqrt{1+\beta^{2}}}, \quad u \in \tilde{N}_{\alpha, \beta} .
$$

Hence, on $\tilde{N}_{\alpha, \beta}$ minimising $\mathcal{W}$ is equivalent to minimising $\mathcal{W}_{h}$. Furthermore, by Lemma 4.5 in [11], in the minimisation process, we can restrict to functions $u \in \tilde{N}_{\alpha, \beta}$ additionally satisfying

$$
u^{\prime}(x)>0 \text { and } 1-\frac{1}{\sqrt{1+u^{\prime}(x)^{2}}} \cosh \left(\frac{\sqrt{1+u^{\prime}(x)^{2}}}{u(x)} x\right) \geq 0 \text { for } x \in(0,1] .
$$

Remark 3 The inequalities in (24) imply

$$
\frac{u(x)}{x} \geq \frac{\sqrt{1+u^{\prime}(x)^{2}}}{\operatorname{arcsinh}\left(u^{\prime}(x)\right)}, \quad x \in(0,1] .
$$

Applying (19) with $y=\operatorname{arcsinh}\left(u^{\prime}(x)\right)$, this gives

$$
u^{\prime}(x) \geq \sinh \left(b_{1}(\alpha(x))\right), \quad \text { where } \quad \alpha(x)=\frac{u(x)}{x}, \quad x \in(0,1] .
$$

Moreover, note that if the second inequality in (24) is strict, then so are the inequalities (25) and (26).

Remark 4 The second inequality in (24) is strict for minimisers $u \in \tilde{N}_{\alpha, \beta}, \beta \in\left[-\alpha,-\sinh \left(b_{1}(\alpha)\right)\right)$ that satisfy (5) (cf. [11, Proof of Proposition 4.18]). For the convenience of the reader we have provided a proof of this statement in the Appendix. 
In what follows we shall make use of the following technical result:

Lemma 8 Let $\alpha>\alpha^{*}$ and $\beta \in\left(-\alpha,-\sinh \left(b_{1}(\alpha)\right)\right]$. For each $u \in \tilde{N}_{\alpha, \beta}$ satisfying (24) and for each $\gamma \in\left[\alpha_{\beta}, \alpha\right)$ there exists a symmetric, strictly positive $v \in C^{1,1}([-1,1])$ such that $v$ satisfies (24), $v( \pm 1)=\gamma, v^{\prime}(-1)=\beta, \alpha \geq v^{\prime}(x)>0$ for $x \in(0,1]$ and $\mathcal{W}(v) \leq \mathcal{W}(u)$. In addition, if u satisfies $u^{\prime} \leq \delta \leq \alpha$ in $(0,1]$ then also the function $v$ satisfies $v^{\prime} \leq \delta \leq \alpha$ in $(0,1]$.

Proof See Corollary 4.20 and its proof in [11]. That result is formulated using the hyperbolic Willmore functional $\mathcal{W}_{h}$ which can be rewritten in terms of $\mathcal{W}$ in view of (23).

Theorem 9 For $\alpha>\alpha^{*}$ and $\beta \in\left(-\alpha,-\sinh \left(b_{1}(\alpha)\right)\right)$ there exists a convex function $u \in \tilde{N}_{\alpha, \beta}$ such that $\mathcal{W}(u)=\inf _{v \in \tilde{N}_{\alpha, \beta}} \mathcal{W}(v)$. The function $u$ belongs to $C^{\infty}([-1,1])$ and solves the Dirichlet boundary value problem (18). Furthermore, u satisfies (24) as well as

$$
u^{\prime}(x) \leq-\beta, \quad x \in[0,1] .
$$

Proof The existence and regularity of a minimizer $u$ satisfying (18), (24) and (27) can be found in [11, Section 4.2.3]. It remains to show that $u$ is convex. Combining (27) with (22) we deduce that

$$
\frac{d}{d x} \frac{u(x)}{x}=\frac{1}{x}\left(u^{\prime}(x)-\frac{u(x)}{x}\right) \leq \frac{1}{x}(-\beta-\alpha)<0, \quad x \in(0,1] .
$$

Next, we claim that $u^{\prime}$ is injective on $[0,1]$. If not, there would exist $x_{1}, x_{2}$ in $(0,1]$ such that $x_{1}<x_{2}$ and $u^{\prime}\left(x_{1}\right)=u^{\prime}\left(x_{2}\right)$. We can choose $x_{1}$ such that $u^{\prime}(x)<u^{\prime}\left(x_{1}\right)$ for all $x \in\left[0, x_{1}\right)$. Let us consider the function $\tilde{u}:[-1,1] \rightarrow \mathbb{R}, \tilde{u}(x)=\frac{u\left(x_{1} x\right)}{x_{1}}$. Clearly $\tilde{u}( \pm 1)=\frac{u\left(x_{1}\right)}{x_{1}}=: \tilde{\alpha}$ and $\tilde{u}^{\prime}(-1)=-u^{\prime}\left(x_{1}\right)=: \tilde{\beta}$. Recalling that $u^{\prime}(x)<u^{\prime}\left(x_{1}\right)$ for $x \in\left[0, x_{1}\right)$ and arguing similarly as in (28) we derive

$$
\tilde{u}^{\prime}(x)=u^{\prime}\left(x_{1} x\right) \leq u^{\prime}\left(x_{1}\right)<\frac{u\left(x_{1}\right)}{x_{1}}=\tilde{\alpha}, \quad x \in[0,1],
$$

so that $\tilde{u} \in \tilde{N}_{\tilde{\alpha}, \tilde{\beta}}$, and $\tilde{\beta}>-\tilde{\alpha}$. On the other hand, (26) computed for $u$ at $x_{1}$ yields $\tilde{\beta} \leq$ $-\sinh \left(b_{1}(\tilde{\alpha})\right)$, and so we have $\tilde{\beta} \in\left(-\tilde{\alpha},-\sinh \left(b_{1}(\tilde{\alpha})\right)\right]$. Evaluating $(25)$ at $x_{2}$ and taking into account that $u^{\prime}\left(x_{1}\right)=u^{\prime}\left(x_{2}\right)=-\tilde{\beta}$ we find

$$
\frac{u\left(x_{2}\right)}{x_{2}} \geq \frac{\sqrt{1+\tilde{\beta}^{2}}}{\operatorname{arcsinh}(-\tilde{\beta})}=\alpha_{\tilde{\beta}} .
$$

Combining this estimate with (28) we infer that

$$
\tilde{\alpha}=\frac{u\left(x_{1}\right)}{x_{1}}>\frac{u\left(x_{2}\right)}{x_{2}} \geq \alpha_{\tilde{\beta}} .
$$

Moreover since $u$ satisfies (24), the same holds for $\tilde{u}$. Lemma 8 with $\alpha=\tilde{\alpha}, \beta=\tilde{\beta}, \gamma=\frac{u\left(x_{2}\right)}{x_{2}}$, and $\delta=u^{\prime}\left(x_{1}\right)$ implies that there exists a symmetric, strictly positive function $\tilde{v} \in C^{1,1}([-1,1])$ satisfying (24), $\tilde{v}( \pm 1)=\frac{u\left(x_{2}\right)}{x_{2}}, \tilde{v}^{\prime}(-1)=-u^{\prime}\left(x_{1}\right), \tilde{v}^{\prime}(x) \leq u^{\prime}\left(x_{1}\right) \leq \alpha$ for $x \in[0,1]$ and $\mathcal{W}(\tilde{v}) \leq \mathcal{W}(\tilde{u})$. Recalling that $u^{\prime}\left(x_{1}\right)=u^{\prime}\left(x_{2}\right)$ we deduce that the function

$$
v(x):= \begin{cases}x_{2} \tilde{v}\left(\frac{x}{x_{2}}\right) & \text { for } x \in\left[-x_{2}, x_{2}\right], \\ u(x) & \text { for } x \in\left[-1,-x_{2}\right) \cup\left(x_{2}, 1\right],\end{cases}
$$

belongs to $\tilde{N}_{\alpha, \beta}$. Furthermore,

$$
\begin{aligned}
\mathcal{W}(v) & =\mathcal{W}(\tilde{v})+4 \pi \int_{x_{2}}^{1} H(x)^{2} u(x) \sqrt{1+u^{\prime}(x)^{2}} d x \\
& \leq \mathcal{W}(\tilde{u})+4 \pi \int_{x_{2}}^{1} H(x)^{2} u(x) \sqrt{1+u^{\prime}(x)^{2}} d x \\
& =\mathcal{W}(u)-4 \pi \int_{x_{1}}^{x_{2}} H(x)^{2} u(x) \sqrt{1+u^{\prime}(x)^{2}} d x .
\end{aligned}
$$


Since $u$ is a mimimum of $\mathcal{W}$ over $\tilde{N}_{\alpha, \beta}$ we deduce that $H \equiv 0$ on $\left[x_{1}, x_{2}\right]$. It is well known that then necessarily $\left.u\right|_{\left[x_{1}, x_{2}\right]}$ is a piece of a catenary (cf. [8, Proposition 5.11]). This however contradicts the fact that $u^{\prime}\left(x_{1}\right)=u^{\prime}\left(x_{2}\right)$. As a consequence, $u^{\prime}$ must be injective on $[0,1]$, so that $u^{\prime}$ is strictly increasing as $u^{\prime}(0)<u^{\prime}(1)$ and we finally infer that $u$ is convex.

Next, we need a result concerning the monotonicity behaviour of $\beta \mapsto \tilde{M}_{\alpha, \beta}$.

Lemma 10 Let $\alpha \underset{\sim}{\sim} \alpha^{*}$ and $\beta, \beta^{\prime} \in\left[-\alpha,-\sinh \left(b_{1}(\alpha)\right)\right)$ with $\beta<\beta^{\prime}$.

Then $\tilde{M}_{\alpha, \beta^{\prime}}<\tilde{M}_{\alpha, \beta}$.

Proof See the Appendix.

Theorem 11 Let $\alpha>\alpha^{*}$. Then there exists a convex function $u \in \tilde{N}_{\alpha,-\alpha}$ such that $\mathcal{W}(u)=$ $\inf _{v \in \tilde{N}_{\alpha,-\alpha}} \mathcal{W}(v)$. The function $u$ belongs to $C^{\infty}([-1,1])$ and solves the Dirichlet boundary value problem (15).

Proof Let $\left(\beta_{k}\right)_{k \in \mathbb{N}}$ be a sequence in $\left(-\alpha,-\sinh \left(b_{1}(\alpha)\right)\right)$ with $\lim _{k \rightarrow \infty} \beta_{k}=-\alpha$. By Theorem 9 , for each $k \in \mathbb{N}$ there exists a convex function $u_{k} \in \tilde{N}_{\alpha, \beta_{k}}$ such that $\mathcal{W}\left(u_{k}\right)=\inf _{v \in \tilde{N}_{\alpha, \beta_{k}}} \mathcal{W}(v)$ and $u_{k}$ is a solution of (18) with $\beta=\beta_{k}$. Obviously we have the uniform bounds

$$
u_{k}(x) \leq \alpha,\left|u_{k}^{\prime}(x)\right| \leq \alpha, x \in[-1,1], k \in \mathbb{N},
$$

while Lemma 10 implies that

$$
\mathcal{W}\left(u_{k}\right)=\tilde{M}_{\alpha, \beta_{k}}<\tilde{M}_{\alpha,-\alpha}, \quad k \in \mathbb{N} .
$$

Furthermore we show in the Appendix that there exists a constant $c_{\alpha}>0$ such that

$$
u_{k}(x) \geq c_{\alpha}, \quad x \in[-1,1], k \in \mathbb{N} .
$$

As a consequence, $\left(u_{k}\right)_{k \in \mathbb{N}}$ is uniformly bounded in $H^{2}(-1,1)$ so that, after possibly extracting a subsequence, there exists $u \in H^{2}(-1,1)$ with

$$
u_{k} \rightarrow u \text { in } H^{2}(-1,1) \text { and } u_{k} \rightarrow u \text { in } C^{1}([-1,1]) .
$$

Clearly, $u$ is strictly positive, symmetric, convex and we have $u( \pm 1)=\alpha, u^{\prime}(-1)=-\alpha$. It is not difficult to see that one can approximate $u$ in $H^{2}(-1,1)$ by a sequence of functions belonging to $\tilde{N}_{\alpha,-\alpha}$ so that we infer

$$
\mathcal{W}(u) \geq \tilde{M}_{\alpha,-\alpha} .
$$

On the other hand, the weak lower semicontinuity of $\mathcal{W}$ together with (30) yields

$$
\mathcal{W}(u) \leq \liminf _{k \rightarrow \infty} \mathcal{W}\left(u_{k}\right) \leq \limsup _{k \rightarrow \infty} \mathcal{W}\left(u_{k}\right) \leq \tilde{M}_{\alpha,-\alpha} \leq \mathcal{W}(u)
$$

so that

$$
\lim _{k \rightarrow \infty} \mathcal{W}\left(u_{k}\right)=\mathcal{W}(u)=\tilde{M}_{\alpha,-\alpha} .
$$

Next, a short calculation together with (32) and (33) shows that

$$
\begin{aligned}
& \frac{\pi}{2} \int_{-1}^{1} \frac{u_{k}(x)}{\left(1+u_{k}^{\prime}(x)^{2}\right)^{\frac{5}{2}}}\left(u_{k}^{\prime \prime}(x)-u^{\prime \prime}(x)\right)^{2} d x \\
& =\mathcal{W}\left(u_{k}\right)+\pi \int_{-1}^{1} \frac{u_{k}^{\prime \prime}(x)}{\left(1+u_{k}^{\prime}(x)^{2}\right)^{\frac{3}{2}}} d x-\frac{\pi}{2} \int_{-1}^{1} \frac{1}{u_{k}(x) \sqrt{1+u_{k}^{\prime}(x)^{2}}} d x \\
& \quad-\pi \int_{-1}^{1} \frac{u_{k}(x) u_{k}^{\prime \prime}(x) u^{\prime \prime}(x)}{\left(1+u_{k}^{\prime}(x)^{2}\right)^{\frac{5}{2}}} d x+\frac{\pi}{2} \int_{-1}^{1} \frac{u_{k}(x) u^{\prime \prime}(x)^{2}}{\left(1+u_{k}^{\prime}(x)^{2}\right)^{\frac{5}{2}}} d x \\
& \rightarrow \mathcal{W}(u)+\pi \int_{-1}^{1} \frac{u^{\prime \prime}(x)}{\left(1+u^{\prime}(x)^{2}\right)^{\frac{3}{2}}} d x \\
& \quad-\frac{\pi}{2} \int_{-1}^{1} \frac{1}{u(x) \sqrt{1+u^{\prime}(x)^{2}}} d x-\frac{\pi}{2} \int_{-1}^{1} \frac{u(x) u^{\prime \prime}(x)^{2}}{\left(1+u^{\prime}(x)^{2}\right)^{\frac{5}{2}}} d x \\
& =0 .
\end{aligned}
$$


With the help of $(29)$ and $(31)$ we infer that $u_{k}^{\prime \prime} \rightarrow u^{\prime \prime}$ in $L^{2}(-1,1)$ so that $\left(u_{k}\right)_{k \in \mathbb{N}}$ converges strongly to $u$ in $H^{2}(-1,1)$. We can use this information in order to establish that $u$ is a smooth solution of (15). Indeed, since $u_{k}$ is a solution of (18) we have

$$
\left\langle\mathcal{W}^{\prime}\left(u_{k}\right), \varphi\right\rangle=0, \quad \varphi \in H_{0}^{2}(-1,1), k \in \mathbb{N},
$$

where the first variation of $\mathcal{W}$ in direction $\varphi \in H_{0}^{2}(-1,1)$ is given by the formula (cf. (29), [12])

$$
\begin{aligned}
\left\langle\mathcal{W}^{\prime}(v), \varphi\right\rangle= & \pi \int_{-1}^{1} \frac{v(x) v^{\prime \prime}(x) \varphi^{\prime \prime}(x)}{\left(1+v^{\prime}(x)^{2}\right)^{\frac{5}{2}}} d x-\frac{5}{2} \pi \int_{-1}^{1} \frac{v(x) v^{\prime}(x) v^{\prime \prime}(x)^{2} \varphi^{\prime}(x)}{\left(1+v^{\prime}(x)^{2}\right)^{\frac{7}{2}}} d x \\
& -\frac{\pi}{2} \int_{-1}^{1} \frac{v^{\prime}(x) \varphi^{\prime}(x)}{v(x)\left(1+v^{\prime}(x)^{2}\right)^{3 / 2}} d x \\
& +\frac{\pi}{2} \int_{-1}^{1}\left(\frac{v^{\prime \prime}(x)^{2} \varphi(x)}{\left(1+v^{\prime}(x)^{2}\right)^{\frac{5}{2}}}-\frac{\varphi(x)}{v(x)^{2}\left(1+v^{\prime}(x)^{2}\right)^{\frac{1}{2}}}\right) d x
\end{aligned}
$$

Letting $k \rightarrow \infty$ we deduce that

$$
\left\langle\mathcal{W}^{\prime}(u), \varphi\right\rangle=0, \quad \varphi \in H_{0}^{2}(-1,1),
$$

and proceeding as in the proof of Theorem 4, Step 2 in [10] we find that $u$ is a smooth solution of (15).

Remark 5 Let us emphasize that the constraint $u^{\prime} \leq \alpha$ in the definition of $\tilde{N}_{\alpha, \beta}$ appears to be crucial in order to construct convex solutions. This observation is based on the results of numerical experiments conducted with the algorithm described in [11, Section 7], which calculates approximate solutions of (18) via the $L^{2}$-gradient flow of $\mathcal{W}$. The corresponding solutions are therefore in general local minima of $\mathcal{W}$ in the class

$$
N_{\alpha, \beta}=\left\{u \in S_{\alpha} \cap C^{1,1}([-1,1]): u^{\prime}(-1)=\beta\right\} .
$$

On the left hand side of Figure 2 we display two solutions for $\alpha=-\beta=3$, while the pictures on the right hand side show their corresponding mean curvature $H$. We remark that the lower solution does not belong to $\tilde{N}_{3,-3}$, and has energy $\mathcal{W} \simeq 0.4172$ while the energy of the upper (convex) solution is $\mathcal{W} \simeq 3.428$. Note also that the values of $H( \pm 1)$ numerically confirm the statement of Theorem 5 .

Proof (Proof of Theorem 2) Fix $\alpha>\alpha^{*}$. We already know that the two catenaries

$$
x \mapsto \frac{\cosh \left(b_{i}(\alpha) x\right)}{b_{i}(\alpha)}, \quad i=1,2,
$$

are solutions of (5)-(6). The existence of a third solution which is convex follows immediately by combining Corollary 6 and Theorem 11. Furthermore, denoting by $\left(u_{k}\right)_{k \in \mathbb{N}}$ the sequence occuring in the proof of Theorem 11 we infer with the help of (30) that $\mathcal{W}\left(u_{k}\right)<\mathcal{W}(u), k \in \mathbb{N}$. Since $u_{k} \in S_{\alpha}$ and $u_{k} \rightarrow u, k \rightarrow \infty$ in $H^{2}(-1,1)$, the function $u$ cannot be a local mimimum of $\mathcal{W}$ in $S_{\alpha}$.

Proof of $(i)$. We claim that the mean curvature $H$ of $u$ is either strictly positive or strictly negative on $(-1,1)$. In view of the symmetry of $H$ it is enough to prove the claim on $[0,1)$. Assume that there exists an $x_{0} \in[0,1)$ such that $H\left(x_{0}\right)=0$. Then there would also exist an $x_{1} \in\left(x_{0}, 1\right)$ such that $H^{\prime}\left(x_{1}\right)=0$, and hence by (16)

$$
C\left(x_{1}+u\left(x_{1}\right) u^{\prime}\left(x_{1}\right)\right)=0 .
$$

As $u^{\prime}(x)>0$ in $(0,1]$ it follows that $C=0$, which upon reinsertion in (16) gives that $H$ is a constant function and hence $H \equiv 0$, which contradicts Corollary 6 . Therefore $H$ is of fixed sign on $(-1,1)$. 

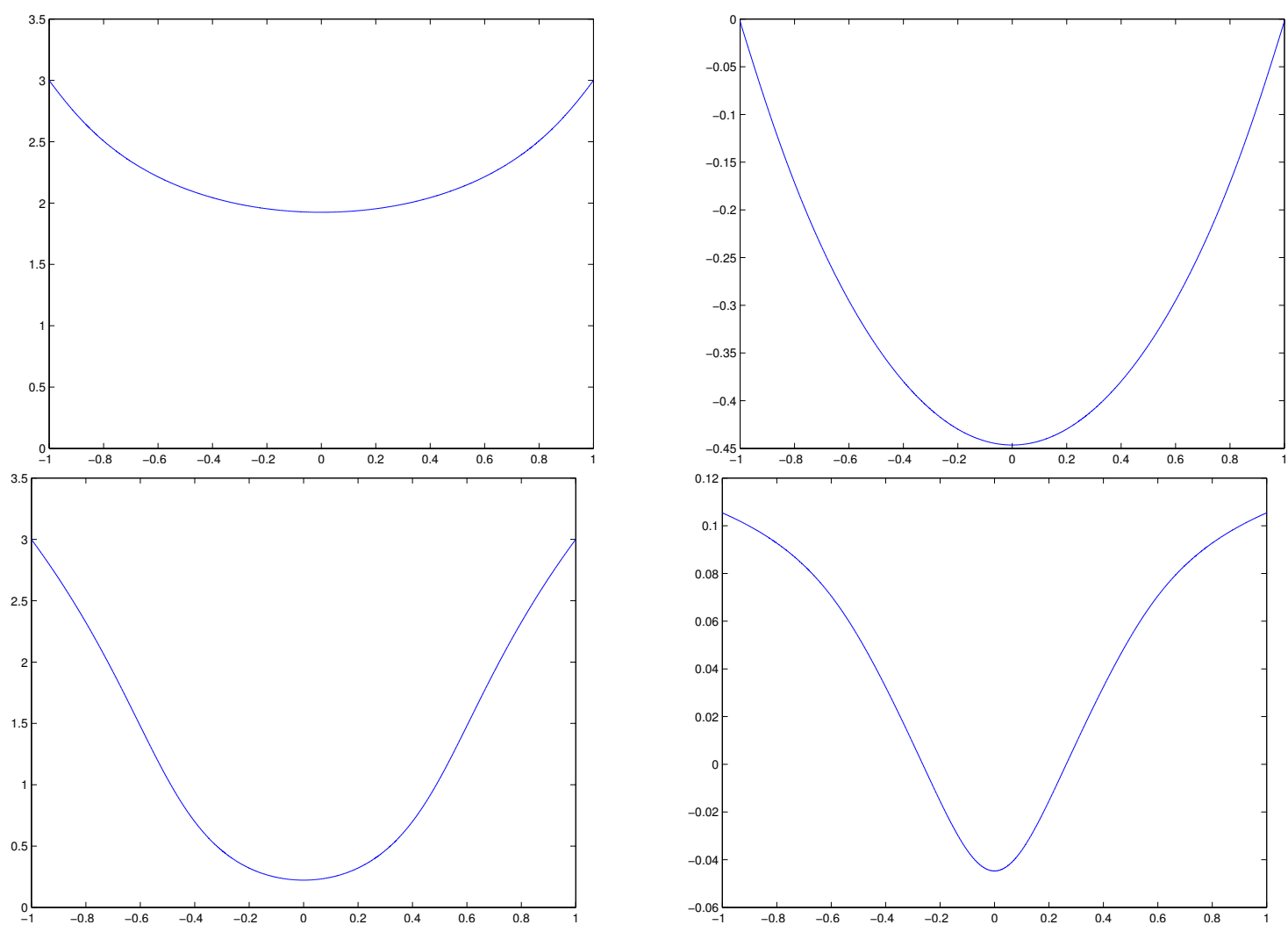

Fig. 2: Numerically computed solutions to (15) (left) and their mean curvature (right) in the case $\alpha=3$.

In order to determine the sign of $H$ we now compute

$$
\begin{array}{rl}
2 \int_{0}^{1} & H(x)\left(1+u^{\prime}(x)^{2}\right)\left(\frac{x u^{\prime}(x)}{u(x)}-1\right) d x \\
& =\int_{0}^{1} \frac{d}{d x}\left(\operatorname{arccosh}\left(\sqrt{1+u^{\prime}(x)^{2}}\right)-x \frac{\sqrt{1+u^{\prime}(x)^{2}}}{u(x)}\right) d x \\
& =\operatorname{arccosh}\left(\sqrt{1+\alpha^{2}}\right)-\frac{\sqrt{1+\alpha^{2}}}{\alpha} \\
& >\operatorname{arccosh}\left(\sqrt{1+\left(\alpha^{*}\right)^{2}}\right)-\frac{\sqrt{1+\left(\alpha^{*}\right)^{2}}}{\alpha^{*}} \\
& =0
\end{array}
$$

since the function $\alpha \mapsto \operatorname{arccosh}\left(\sqrt{1+\alpha^{2}}\right)-\frac{\sqrt{1+\alpha^{2}}}{\alpha}$ is strictly increasing. As $u \in \tilde{N}_{\alpha,-\alpha}$, we have $u^{\prime}(x) \leq \alpha$ which together with $(22)$ yields

$$
\frac{x u^{\prime}(x)}{u(x)} \leq 1 \quad \text { for } x \in[0,1]
$$

Assume that $H>0$ on $(-1,1)$. Combining (34) and (35) shows

$$
0 \geq 2 \int_{0}^{1} H(x)\left(1+u^{\prime}(x)^{2}\right)\left(\frac{x u^{\prime}(x)}{u(x)}-1\right) d x>0
$$

which is impossible. Hence $H(x)<0$ for all $x \in(-1,1)$, which proves $(i)$. 
Proof of (ii). We are now in a position to show that $u$ and $H$ are strictly convex. Strict convexity of $u$ follows immediately by solving (3) for $u^{\prime \prime}$ and using $H(x)<0$ :

$$
u^{\prime \prime}(x)=-2 H(x)\left(1+u^{\prime}(x)^{2}\right)^{\frac{3}{2}}+\frac{1+u^{\prime}(x)^{2}}{u(x)}>0, \quad x \in[-1,1] .
$$

In order to see that $H$ is strictly convex, we first observe that evaluating (9) at the origin gives

$$
C=-u(0) H(0)^{2}+H(0)<0 .
$$

Differentiating (16) and using (35) (which, due to the symmetry of $u$, is valid in $[-1,1]$ ) as well as $(i)$, it follows for all $x \in(-1,1)$ that

$$
\begin{aligned}
H^{\prime \prime}(x) & =(-C)\left(\frac{1}{u(x)^{2}}-2 \frac{x u^{\prime}(x)}{u(x)^{3}}+\frac{u^{\prime \prime}(x)}{u(x)}-\frac{u^{\prime}(x)^{2}}{u(x)^{2}}\right) \\
& \geq(-C)\left(-\frac{1+u^{\prime}(x)^{2}}{u(x)^{2}}+\frac{u^{\prime \prime}(x)}{u(x)}\right) \\
& =(-C)\left(-\frac{1+u^{\prime}(x)^{2}}{u(x)^{2}}-2 H(x) \frac{\left(1+u^{\prime}(x)^{2}\right)^{\frac{3}{2}}}{u(x)}+\frac{1+u^{\prime}(x)^{2}}{u(x)^{2}}\right) \\
& =2 C H(x) \frac{\left(1+u^{\prime}(x)^{2}\right)^{\frac{3}{2}}}{u(x)} \\
& >0 .
\end{aligned}
$$

Proof of (iii). Let us first show that

$$
u(x)>u_{b_{2}}(x)=\frac{1}{b_{2}} \cosh \left(b_{2} x\right), \quad x \in(-1,1),
$$

where $b_{2}=b_{2}(\alpha)$. Since

$$
\frac{\alpha}{\sqrt{1+\alpha^{2}}}-u(0)=\int_{0}^{1} \frac{d}{d x}\left[\frac{u(x)}{\sqrt{1+u^{\prime}(x)^{2}}}\right] d x=2 \int_{0}^{1} u(x) u^{\prime}(x) H(x) d x<0
$$

by $(i)$, and as $\alpha<\sinh \left(b_{2}\right)$, this implies

$$
u(0)>\frac{\alpha}{\sqrt{1+\alpha^{2}}}>\frac{\alpha}{\cosh \left(b_{2}\right)}=\frac{1}{b_{2}}=u_{b_{2}}(0),
$$

so that (36) holds at the origin. Set $x^{*}=\inf \left\{x \in[0,1] \mid u(x)=u_{b_{2}}(x)\right\}$. Since $(36)$ holds at $x=0$, we have $x^{*} \in(0,1]$. Assume that $x^{*}<1$. Clearly

$$
u^{\prime}\left(x^{*}\right) \leq u_{b_{2}}^{\prime}\left(x^{*}\right)
$$

Since $u\left(x^{*}\right)=u_{b_{2}}\left(x^{*}\right)$ we deduce with the help of $(22)$

$$
\frac{\cosh \left(b_{2} x^{*}\right)}{b_{2} x^{*}}=\frac{u\left(x^{*}\right)}{x^{*}} \geq \alpha
$$

so that by (19) $b_{2} x^{*} \leq b_{1}$ or $b_{2} x^{*} \geq b_{2}$. Since $x^{*}<1$ and $b_{1}<b_{0}$, this in particular implies $b_{2} x^{*}<b_{0}$. By Theorem 11, $u$ is a minimiser in $\tilde{N}_{\alpha,-\alpha}$. Remark 4 followed by Remark 3 thus yields that

$$
u^{\prime}\left(x^{*}\right)>\sinh \left(b_{1}\left(\frac{u\left(x^{*}\right)}{x^{*}}\right)\right)=\sinh \left(b_{1}\left(\frac{\cosh \left(b_{2} x^{*}\right)}{b_{2} x^{*}}\right)\right)=\sinh \left(b_{2} x^{*}\right)=u_{b_{2}}^{\prime}\left(x^{*}\right)
$$

by the definition of $b_{1}$ and since $b_{2} x^{*}<b_{0}$. This contradicts (37) so that $x^{*}=1$, proving (36) on $[0,1)$. The claim on $(-1,1)$ follows by the symmetry of $u$ and $u_{b_{2}}$. 
Let us finally show that

$$
u(x)<u_{b_{1}}(x)=\frac{1}{b_{1}} \cosh \left(b_{1} x\right), \quad x \in(-1,1),
$$

where $b_{1}=b_{1}(\alpha)$. Set $x^{*}=\sup \left\{x \in[0,1] \mid u^{\prime}(x)=u_{b_{1}}^{\prime}(x)\right\}$. Clearly $x^{*}<1$ and $u^{\prime}(x)>u_{b_{1}}^{\prime}(x)$ for $x \in\left(x^{*}, 1\right]$. Let us assume that $x^{*}>0$. We have $u^{\prime}\left(x^{*}\right)=u_{b_{1}}^{\prime}\left(x^{*}\right)=\sinh \left(b_{1} x^{*}\right)$ and

$$
u\left(x^{*}\right)-u_{b_{1}}\left(x^{*}\right)=\int_{x^{*}}^{1}\left(u_{b_{1}}^{\prime}(x)-u^{\prime}(x)\right) d x<0 .
$$

Therefore,

$$
\frac{u\left(x^{*}\right)}{x^{*}}<\frac{u_{b_{1}}\left(x^{*}\right)}{x^{*}}=\frac{\cosh \left(b_{1} x^{*}\right)}{b_{1} x^{*}}=\frac{\sqrt{1+u^{\prime}\left(x^{*}\right)^{2}}}{\operatorname{arcsinh}\left(u^{\prime}\left(x^{*}\right)\right)} .
$$

On the other hand (25) computed in $x^{*}$ yields

$$
\frac{u\left(x^{*}\right)}{x^{*}} \geq \frac{\sqrt{1+u^{\prime}\left(x^{*}\right)^{2}}}{\operatorname{arcsinh}\left(u^{\prime}\left(x^{*}\right)\right)},
$$

a contradiction. Hence $x^{*}=0$ which implies that $u^{\prime}(x)>u_{b_{1}}^{\prime}(x)$ for $x \in(0,1]$. Integration yields (38) on $[0,1)$, and the symmetry of $u$ and $u_{b_{1}}$ gives $(38)$ on $(-1,1)$.

This completes the proof of Theorem 2 .

\section{Appendix}

Proof of Remark 4. If the second inequality in (24) were not strict, then there would exist an $x_{1} \in(0,1)$ such that

$$
u\left(x_{1}\right)=\frac{u\left(x_{1}\right)}{\sqrt{1+u^{\prime}\left(x_{1}\right)^{2}}} \cosh \left(\frac{\sqrt{1+u^{\prime}\left(x_{1}\right)^{2}}}{u\left(x_{1}\right)} x_{1}\right) .
$$

Let

$$
u_{\gamma}(x)=\frac{1}{\gamma} \cosh (\gamma x), \quad \text { where } \quad \gamma=\frac{\sqrt{1+u^{\prime}\left(x_{1}\right)^{2}}}{u\left(x_{1}\right)} .
$$

Note that $u\left(x_{1}\right)=u_{\gamma}\left(x_{1}\right)$ and

$$
u_{\gamma}^{\prime}\left(x_{1}\right)=\sinh \left(\gamma x_{1}\right)=\sqrt{\cosh ^{2}\left(\gamma x_{1}\right)-1}=u^{\prime}\left(x_{1}\right),
$$

where we used (39) for the last equality. Since $u \in \tilde{N}_{\alpha, \beta}$ this implies $u_{\gamma}^{\prime}(x) \leq u_{\gamma}^{\prime}\left(x_{1}\right)=u^{\prime}\left(x_{1}\right) \leq \alpha$ for $x \in\left[0, x_{1}\right]$. As a consequence the function

$$
v(x):= \begin{cases}u_{\gamma}(x) & \text { for } x \in\left[-x_{1}, x_{1}\right] \\ u(x) & \text { otherwise }\end{cases}
$$

belongs to $\tilde{N}_{\alpha, \beta}$ and $\mathcal{W}(v) \leq \mathcal{W}(u)$. Since $u$ is a minimiser of $\mathcal{W}$ in $\tilde{N}_{\alpha, \beta}$ we infer that $\mathcal{W}(v)=\mathcal{W}(u)$ and hence $H[u] \equiv 0$ on $\left[-x_{1}, x_{1}\right]$. It follows from $\left[8\right.$, Proposition 5.11] and the boundary values that $u \equiv u_{\gamma}$ on $\left[-x_{1}, x_{1}\right]$. Since both $u$ and $u_{\gamma}$ solve $(5)$ on $(-1,1)$ we obtain $u \equiv u_{\gamma}$ on $[-1,1]$ which is in contradiction with $\beta \in\left[-\alpha,-\sinh \left(b_{1}(\alpha)\right)\right)$.

Proof of Lemma 10. Here we essentially follow the argument of Lemma 3.11 in [2] with minor modifications. Let us first prove that

$$
\tilde{M}_{\alpha, \beta^{\prime}}<\tilde{M}_{\alpha, \beta} \quad \text { for all } \beta, \beta^{\prime} \in\left(-\alpha,-\sinh \left(b_{1}(\alpha)\right)\right) \text { with } \beta<\beta^{\prime} .
$$

We observe that (21) implies $\alpha>\alpha_{\beta}$ and $\alpha>\alpha_{\beta^{\prime}}$. By Theorem 9 there exists a convex function $u \in \tilde{N}_{\alpha, \beta}$ satisfying (24) such that $\mathcal{W}(u)=\inf _{v \in \tilde{N}_{\alpha, \beta}} \mathcal{W}(v)$. Since $u^{\prime}(1)=-\beta>-\beta^{\prime}$ there is $x^{*} \in(0,1)$ with $u^{\prime}\left(x^{*}\right)=-\beta^{\prime}$. The function $\tilde{u}:[-1,1] \rightarrow \mathbb{R}, \tilde{u}(x):=\frac{1}{x^{*}} u\left(x^{*} x\right)$ then satisfies $(24), \tilde{u}( \pm 1)=\frac{1}{x^{*}} u\left(x^{*}\right)=: \tilde{\alpha}$ and $\tilde{u}^{\prime}(-1)=\beta^{\prime}$. In view of (22) we have $\tilde{\alpha} \geq \alpha$. Furthermore, since $u$ is convex we infer that

$$
\tilde{u}^{\prime}(x)=u^{\prime}\left(x^{*} x\right) \leq u^{\prime}\left(x^{*}\right) \leq \alpha \leq \tilde{\alpha} \quad \text { in }[0,1]
$$

so that $\tilde{u} \in \tilde{N}_{\tilde{\alpha}, \beta^{\prime}}$. Evaluating (26) at $x^{*}$ implies $\beta^{\prime} \leq-\sinh \left(b_{1}(\tilde{\alpha})\right)$. Since $\beta^{\prime}>-\alpha \geq-\tilde{\alpha}$, we therefore have $\beta^{\prime} \in\left(-\tilde{\alpha},-\sinh \left(b_{1}(\tilde{\alpha})\right)\right]$. As observed earlier, $\alpha>\alpha_{\beta^{\prime}}$ and so $\alpha \in\left(\alpha_{\beta^{\prime}}, \tilde{\alpha}\right]$. If $\tilde{\alpha}>\alpha$, Lemma 8 yields the existence of 
a symmetric, strictly positive function $v \in C^{1,1}([-1,1])$ satisfying $v( \pm 1)=\alpha, v^{\prime}(-1)=\beta^{\prime}, v^{\prime} \leq \alpha$ in $[0,1],(24)$ and $\mathcal{W}(v) \leq \mathcal{W}(\tilde{u})$. If $\tilde{\alpha}=\alpha$ we simply choose $v=\tilde{u}$. In any case we have $v \in \tilde{N}_{\alpha, \beta^{\prime}}$ and

$$
\begin{aligned}
\mathcal{W}(u) & =4 \pi \int_{0}^{x^{*}} H(x)^{2} u(x) \sqrt{1+u^{\prime}(x)^{2}} d x+4 \pi \int_{x^{*}}^{1} H(x)^{2} u(x) \sqrt{1+u^{\prime}(x)^{2}} d x \\
& =\mathcal{W}(\tilde{u})+4 \pi \int_{x^{*}}^{1} H(x)^{2} u(x) \sqrt{1+u^{\prime}(x)^{2}} d x \\
& \geq \mathcal{W}(v)+4 \pi \int_{x^{*}}^{1} H(x)^{2} u(x) \sqrt{1+u^{\prime}(x)^{2}} d x \\
& \geq \tilde{M}_{\alpha, \beta^{\prime}}+4 \pi \int_{x^{*}}^{1} H(x)^{2} u(x) \sqrt{1+u^{\prime}(x)^{2}} d x .
\end{aligned}
$$

We observe that $H \not \equiv 0$ on $\left[x^{*}, 1\right]$, for otherwise $u_{\mid\left[x^{*}, 1\right]}$ would have to be a catenary, contradicting the fact that $u(1)=\alpha, u^{\prime}(1)=-\beta$ and $(20)$. Recalling that $\mathcal{W}(u)=\tilde{M}_{\alpha, \beta}$ we infer $(40)$.

In order to complete the proof of Lemma 10 it is in view of (40) sufficient to show that

$$
\tilde{M}_{\alpha, \beta} \leq \tilde{M}_{\alpha,-\alpha} \quad \text { for all } \beta \in\left(-\alpha,-\sinh \left(b_{1}(\alpha)\right)\right)
$$

Fix $\beta \in\left(-\alpha,-\sinh \left(b_{1}(\alpha)\right)\right)$ and let $u \in \tilde{N}_{\alpha,-\alpha}$ be an arbitrary function satisfying (24). Note that (21) implies $\alpha>\alpha \beta$ Since $u^{\prime}(1)=\alpha>-\beta$ there is an $x^{*} \in(0,1)$ such that $u^{\prime}\left(x^{*}\right)=-\beta$ and $0<u^{\prime}(x)<u^{\prime}\left(x^{*}\right), x \in\left(0, x^{*}\right)$. The function $\tilde{u}(x)=\frac{u\left(x^{*} x\right)}{x^{*}}$ then satisfies $(24), \tilde{u}( \pm 1)=\frac{u\left(x^{*}\right)}{x^{*}}=: \tilde{\alpha}$ and $\tilde{u}^{\prime}(-1)=\beta$. In view of $(22)$ we have $\tilde{\alpha} \geq \alpha$ and therefore $\tilde{u}^{\prime}(x) \leq u^{\prime}\left(x^{*}\right)=-\beta<\alpha \leq \tilde{\alpha}$ for $x \in[0,1]$, so that $\tilde{u} \in \tilde{N}_{\tilde{\alpha}, \beta}$. Similarly as above we deduce from (21), (25), (26) that $\beta \in\left(-\tilde{\alpha},-\sinh \left(b_{1}(\tilde{\alpha})\right)\right]$ as well as $\tilde{\alpha} \geq \alpha>\alpha_{\beta}$. Choosing $v=\tilde{u}$ if $\alpha=\tilde{\alpha}$, and $v$ according to Lemma 8 if $\tilde{\alpha}>\alpha$, we obtain a symmetric, strictly positive function $v \in C^{1,1}([-1,1])$ satisfying $v( \pm 1)=\alpha, v^{\prime}(-1)=\beta, v^{\prime} \leq \alpha$ in $[0,1],(24)$ and $\mathcal{W}(v) \leq \mathcal{W}(\tilde{u})$. As a result

$$
\mathcal{W}(u) \geq \mathcal{W}(\tilde{u}) \geq \mathcal{W}(v) \geq \tilde{M}_{\alpha, \beta},
$$

from which we deduce (41). This finishes the proof of Lemma 10

Proof of (31). We use the argument from the proof of Lemma 4.9 in [11]. For the convenience of the reader we reproduce the relevant calculations and adapt them to our particular situation. To begin,

$$
\begin{aligned}
\mathcal{W}\left(u_{k}\right) & =\pi \int_{0}^{1}\left(-\frac{u_{k}^{\prime \prime}(x)}{\left(1+u_{k}^{\prime}(x)^{2}\right)^{\frac{3}{2}}}+\frac{1}{u_{k}(x) \sqrt{1+u_{k}^{\prime}(x)^{2}}}\right)^{2} u_{k}(x) \sqrt{1+u_{k}^{\prime}(x)^{2}} d x \\
& \geq \pi \int_{0}^{1} \frac{1}{u_{k}(x) \sqrt{1+u_{k}^{\prime}(x)^{2}}} d x-2 \pi \int_{0}^{1} \frac{u_{k}^{\prime \prime}(x)}{\left(1+u_{k}^{\prime}(x)^{2}\right)^{\frac{3}{2}}} d x \\
& =\pi \int_{0}^{1} \frac{1}{u_{k}(x) \sqrt{1+u_{k}^{\prime}(x)^{2}}} d x+2 \pi \frac{\beta_{k}}{\sqrt{1+\beta_{k}^{2}}} .
\end{aligned}
$$

Since $u_{k}^{\prime} \leq \alpha$ in $[0,1]$ we have $u_{k}(x) \leq u_{k}(0)+\alpha x, x \in[0,1]$ and therefore

$$
\frac{\pi}{\sqrt{1+\alpha^{2}}} \int_{0}^{1} \frac{1}{u_{k}(0)+\alpha x} d x \leq \mathcal{W}\left(u_{k}\right)+2 \pi \frac{\left|\beta_{k}\right|}{\sqrt{1+\beta_{k}^{2}}} \leq \tilde{M}_{\alpha,-\alpha}+2 \pi \frac{\alpha}{\sqrt{1+\alpha^{2}}}
$$

as $\left|\beta_{k}\right| \leq \alpha$. Solving this inequality for $u_{k}(0)$ and recalling that $u_{k}$ is convex we infer

$$
\inf _{x \in[0,1]} u_{k}(x)=u_{k}(0) \geq \frac{\alpha}{\exp \left(\frac{\alpha \sqrt{1+\alpha^{2}}}{\pi} \tilde{M}_{\alpha,-\alpha}+2 \alpha^{2}\right)-1},
$$

which proves (31).

Acknowledgements The authors thank Friedhelm Schieweck for his help with the numerical experiments. Financial support for Glen Wheeler from the Alexander-von-Humboldt Stiftung and for Anna Dall'Acqua and Klaus Deckelnick from the Deutsche Forschungsgemeinschaft (DFG) for the project "Randwertprobleme für Willmoreflächen - Analysis, Numerik und Numerische Analysis" (DE 611/5.1) is gratefully acknowledged. 


\section{References}

1. M. Bauer and E. Kuwert. Existence of minimizing Willmore surfaces of prescribed genus. Int. Math. Res. Not., 2003(10):553-576, 2003.

2. M. Bergner, A. Dall'Acqua, and S. Fröhlich. Symmetric Willmore surfaces of revolution satisfying natural boundary conditions. Calc. Var. Partial Differential Equations, 39(3-4):361-378, 2010.

3. M. Bergner, A. Dall'Acqua, and S. Fröhlich. Willmore surfaces of revolution with two prescribed boundary circles. J. Geom. Anal., 2012. On-line first.

4. Y. Bernard and T. Riviere. Local Palais-Smale sequences for the Willmore functional. Comm. Anal. Geom., 19(3):563-600, 2011.

5. W. Blaschke. Vorlesungen über Differentialgeometrie. Vol. I-III, 1929

6. R. Bryant and P. Griffiths. Reduction for constrained variational problems and $\int \frac{1}{2} k^{2} d s$. Amer. J. Math., 108(3):525-570, 1986

7. R.L. Bryant. A duality theorem for Willmore surfaces. J. Differential Geom., 20(1):23-53, 1984.

8. B. Dacorogna. Introduction to the Calculus of Variations. Imperial College Press, 2004.

9. A. Dall'Acqua. Uniqueness for the homogeneous Dirichlet Willmore boundary value problem. Ann. Glob. Anal. Geom. On-line first.

10. A. Dall'Acqua, K. Deckelnick, and H.-Ch. Grunau. Classical solutions to the Dirichlet problem for Willmore surfaces of revolution. Adv. Calc. Var., 1(4):379-397, 2008.

11. A. Dall'Acqua, S. Fröhlich, H.-Ch. Grunau, and F. Schieweck. Symmetric Willmore surfaces of revolution satisfying arbitrary Dirichlet boundary data. Adv. Calc. Var., 4(1):1-81, 2011.

12. K. Deckelnick and H.-Ch. Grunau. A Navier boundary value problem for Willmore surfaces of revolution. Analysis, 29(3):229-258, 2009

13. H.-Ch. Grunau. The asymptotic shape of a boundary layer of symmetric willmore surfaces of revolution. In C. et al. (eds) Bandle, editor, Inequalities and Applications 2010, volume 161 of International Series of Numerical Mathematics, pages 19-29. Springer Basel, 2012.

14. U. Hertrich-Jeromin and U. Pinkall. Ein Beweis der Willmoreschen Vermutung für Kanaltori. J. Reine Angew. Math., 430:21-34, 1992

15. E. Kuwert. The Willmore functional. Lectures held at ETH in 2007.

16. E. Kuwert and Y. Li. $W^{2,2}$-conformal immersions of a closed Riemann surface into $\mathbb{R}^{n}$. Arxiv preprint arXiv:1007.3967, 2010.

17. E. Kuwert and R. Schätzle. Closed surfaces with bounds on their Willmore energy. To appear in Annali Sc. Norm. Sup. Pisa.

18. J. Langer and D.A. Singer. Curve straightening and a minimax argument for closed elastic curves. Topology, $24(1): 75-88,1985$.

19. J.C.C. Nitsche. Boundary value problems for variational integrals involving surface curvatures. Quart. Appl. Math, 51(2):363-387, 1993.

20. B. Palmer. The conformal Gauss map and the stability of Willmore surfaces. Ann. Global Anal. Geom., 9(3):305$317,1991$.

21. B. Palmer. Uniqueness theorems for Willmore surfaces with fixed and free boundaries. Indiana Univ. Math. J., 49(4):1581-1602, 2000.

22. T. Rivière. Analysis aspects of Willmore surfaces. Invent. Math., 174(1):1-45, 2008.

23. R. Schätzle. The Willmore boundary problem. Calc. Var. Partial Differential Equations, 37(3-4):275-302, 2010.

24. L. Simon. Existence of surfaces minimizing the Willmore functional. Comm. Anal. Geom., 1(2):281-326, 1993.

25. M. Struwe. Plateau's problem and the calculus of variations. Math. Notes 35, Princeton University Press, Princeton, 1989.

26. G. Thomsen. Über konforme Geometrie I: Grundlagen der konformen Flächentheorie. (German). Abh. Math. Sem. Hamburg, 3:31-56, 1923.

27. J.L. Weiner. On a problem of Chen, Willmore, et al. Indiana Univ. Math. J., 27(1):19-35, 1978.

28. T.J. Willmore. Riemannian geometry. Oxford Science Publications. The Clarendon Press Oxford University Press, New York, 1993. 